\title{
(C) OPEN ACCESS \\ Socioeconomic inequalities in pregnancy outcome associated with Down syndrome: a population-based study
}

\author{
Judith L S Budd, Elizabeth S Draper, Robyn R Lotto, Laura E Berry, Lucy K Smith
}

Department of Health Sciences, University of Leicester, Leicester, UK

\section{Correspondence to} Dr Lucy K Smith, Department of Health Sciences, University of Leicester, 22-28 Princess Road West, Leicester LE1 6TP, UK; Iks1@le.ac.uk

Received 2 July 2014 Revised 14 April 2015 Accepted 14 April 2015 Published Online First 12 June 2015

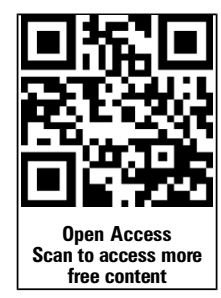

CrossMark

To cite: Budd JLS, Draper ES, Lotto RR, et al. Arch Dis Child Fetal Neonatal Ed 2015;100 F400-F404.

\section{ABSTRACT}

Objective To investigate socioeconomic inequalities in outcome of pregnancy associated with Down syndrome (DS) compared with other congenital anomalies screened for during pregnancy.

Design and setting Retrospective population-based registry study (East Midlands \& South Yorkshire in England).

Participants All registered cases of DS and nine selected congenital anomalies with poor prognostic outcome (the UK Fetal Anomaly Screening Programme (FASP)9) with an end of pregnancy date between 1 January 1998 and 31 December 2007. Main outcome measures: Poisson regression models were used to explore outcome measures, including socioeconomic variation in rates of anomaly; antenatal detection; pregnancy outcome; live birth incidence and neonatal mortality. Deprivation was measured using the Index of Multiple Deprivation 2004 at super output area level.

Results There were 1151 cases of DS and 1572 cases of the nine severe anomalies combined. The overall rate of antenatal detection was $57 \%$ for DS, which decreased with increasing deprivation (rate ratio comparing the most deprived tenth with the least deprived: 0.76 (0.60 to 0.97)). Antenatal detection rates were considerably higher for FASP9 anomalies (86\%), with no evidence of a trend with deprivation $(0.9995 \%$ $\mathrm{Cl}(0.84$ to 1.17$))$. The termination of pregnancy rate following antenatal diagnosis was higher for DS (86\%) than the FASP9 anomalies (70\%). Both groups showed wide socioeconomic variation in the termination of pregnancy rate (rate ratio: DS: 0.76 (0.58 to 0.99); FASP9 anomalies: 0.80 (0.65 to 0.97$)$ ). Consequently, socioeconomic inequalities in live birth and neonatal mortality rates associated with these anomalies arise that were not observed in utero.

Conclusions Socioeconomic inequalities exist in the antenatal detection of DS, and subsequent termination rates are much higher for DS than other anomalies. Termination rates for all anomalies are lower in more deprived areas leading to wide socioeconomic inequalities in live born infants with a congenital anomaly, particularly DS, and subsequent neonatal mortality.

\section{INTRODUCTION}

Although only $2-3 \%$ of UK pregnancies are affected by congenital anomalies, they impact disproportionately on mortality, with almost $30 \%$ of neonatal and infant deaths attributable to anomaly causes. ${ }^{12}$ Consequently, primary preventative measures have become integral to antenatal care,

\section{What is already known on this topic?}

Rates of antenatal detection of nine nationally audited anomalies with a poor prognostic outcome are similar for all deprivation groups.

- Variations in rates of termination of pregnancy have led to socioeconomic inequalities in the rates of live birth associated with congenital anomaly and neonatal mortality.

- Little is known about the impact of socioeconomic deprivation on antenatal detection and termination of pregnancy for Down syndrome (DS).

\section{What this study adds?}

Rates of antenatal detection of DS are much lower than for other anomalies and are lower in more deprived areas.

- Rates of termination of pregnancy are higher for DS than other anomalies, but are significantly lower among women from more deprived areas.

- The combination of these factors results in extremely wide socioeconomic inequalities in relation to birth with congenital anomalies, especially DS, and subsequent mortality.

together with considerable investment in antenatal screening, resulting in the implementation of the UK Fetal Anomaly Screening Programme (FASP) in 2010. ${ }^{3}$ This aims to identify 11 anomalies: serious cardiac, anencephaly, trisomies 13 and 18, spina bifida, exomphalos, renal agenesis, lethal skeletal dysplasia, congenital diaphragmatic hernia, cleft lip and gastroschisis. The first nine present an extremely poor prognosis (FASP9), while cleft lip and gastroschisis require early surgical intervention. All are detectable by antenatal ultrasound, with screening offered to women between $18^{+0}$ and $20^{+6}$ weeks' gestation. The detection of a potentially lethal fetal anomaly permits the option of termination of pregnancy.

Previous examination of the FASP9 anomalies found no difference in occurrence rates in utero or antenatal detection by socioeconomic deprivation. ${ }^{4}$ However there were lower rates of termination demonstrated in areas of higher deprivation. The 
consequent outcome is variation, with socioeconomic deprivation, in the number of live born infants with one of these anomalies, which, together with associated mortality and morbidity, offers an explanation for the inequalities seen nationally. ${ }^{4}$

Antenatal detection of Down syndrome (DS) in England differs, since a separate national screening system exists and additional methods of diagnosis are employed. While associated soft markers may be suspected during the initial booking scan, the majority of cases have historically been diagnosed following later uptake of blood tests or more invasive procedures. Cases of DS outnumber each of the FASP anomalies and are associated with a high rate of pregnancy termination. ${ }^{5}$

Congenital anomaly data are collated by a number of regional registers, permitting consideration of inequalities in outcome from antenatal diagnosis through to the end of pregnancy and neonatal mortality associated with anomalies. Registers cover only $34 \%$ of England and all of Wales, with significant geographical areas, including London and the South-East, lacking any form of routine surveillance. This has implications not only for monitoring trends in the occurrence of anomalies, but also in service planning.

Data from a large English congenital anomaly register ( 10\% of births in England) are used here to explore relative socioeconomic inequalities in DS and the FASP9 anomalies.

\section{METHODS}

The population-based East Midlands and South Yorkshire Congenital Anomalies Register (EMSYCAR) records congenital anomalies in fetuses and infants of mothers living within the region at the time of delivery, monitoring approximately 60000 births annually (excluding Northamptonshire, which joined in 2002). Data on live births, spontaneous fetal losses and stillbirths (before or after 24 weeks' gestation), and terminations of affected pregnancies at any gestation were provided, using multiple sources of case ascertainment from the care pathway, including prenatal ultrasound and screening, delivery reports, birth notifications, pathology, cytogenetics, clinical genetics, neonatal medicine and paediatric surgery. All fetuses registered with either DS (trisomy 21) or one of the FASP9 anomalies in pregnancies ending between 1 January 1998 and 31 December 2007 were included.

FASP9 anomalies included only those conditions where the International Classification of Diseases (ICD)-10 code uniquely identified an anomaly with a high degree of certainty of an adverse prognosis; two chromosomal (trisomies 13 and 18) and seven non-chromosomal anomalies (anencephaly, spina bifida, hypoplastic left heart, bilateral renal agenesis, lethal skeletal dysplasia, diaphragmatic hernia and exomphalos) met this criterion. Where fetuses were registered with a chromosomal diagnosis, coexisting anomalies were deemed secondary to the underlying problem. The definition of 'antenatally detected' followed European Congenital Anomaly Surveillance System (EUROCAT) guidelines, determined by the earliest suggestion of the precise, or closely related, anomaly, or identification of an antenatal soft marker 'nearly $100 \%$ predictive' of the anomaly present at delivery. The presence of a soft marker alone (eg, 'raised nuchal translucency') is insufficient evidence of DS; however, DS can be considered antenatally detected using EUROCAT guidelines in the absence of invasive testing if multiple clinical signs are present, and termination of pregnancies are indeed performed on this basis. Similarly, FASP9 anomalies can be considered present based on clinical findings without a postmortem result.

Live birth denominators were obtained from the Office for National Statistics and stillbirths from the Centre for Maternal and Child Enquiries (CMACE) by year of birth, mother's age and tenth of deprivation of mother's residence at delivery. Deprivation was measured by the Index of Multiple Deprivation $2004^{6}$ comprising seven domain indices at the super output area level (income, employment, health and disability, education, skills and training, housing and services, living environment and crime). Super output areas are the smallest areas for which these deprivation data are available, their small size (1500 residents) limiting the heterogeneity within them. All super output areas in England were ranked by deprivation score and divided into 10 groups with roughly equal numbers of births, from 1 (least) to 10 (most deprived). The mother's postcode at delivery was located within a super output area and the deprivation score for this area was applied to her. Analyses were undertaken at the individual case (fetus or baby) level. Poisson regression models ${ }^{7}$ were used to assess trends in the six outcome measures by deprivation tenth before and after adjustment for year of birth and maternal age:

1. Rate of anomalies in utero (all cases of selected anomalies whenever identified) (denominator: total live births, still births and known late fetal losses and terminations of pregnancy with a fetal anomaly)

2. Rate of antenatal detection (denominator: all cases of anomalies)

3. Rate of termination of pregnancy for anomaly (denominator: antenatally detected cases)

4. Rate of fetal loss or stillbirth with an anomaly (denominator: total live births, stillbirths and known late fetal losses registered with a fetal anomaly)

5. Rate of live births with an anomaly (denominator: total live births)

6. Rate of neonatal mortality of infants with an anomaly (denominator: total live births).

Models were fitted for DS versus all FASP9 anomalies combined. Models were then fitted for the FASP9 chromosomal and non-chromosomal anomalies separately. Interactions were explored to assess the change in the effect of deprivation over time. We included maternal age (grouped <20, 20-24, 25-29, $30-34,>35$ years) in the models to assess its influence on any observed socioeconomic inequality. Two-sided significance testing was used. Confidence limits were obtained with the delta method. ${ }^{8}$ The effect of gestational age at detection of anomaly on termination of pregnancy was also assessed $(<17,18-21$, 22-23, >24 weeks' gestation).

\section{RESULTS}

Between 1998 and 2007, there were 1151 registrations of DS with full postcode and maternal age information, and 1695 further registrations of FASP9 anomalies, representing 1472 fetuses with a single FASP9 anomaly and 107 fetuses with two or more, making 1579 fetuses in total. Of these, seven also had DS and were included only in that group, leaving 1572 cases.

Overall antenatal detection rates (table 1 ) were much lower for DS $(57 \%)$ than the FASP9 anomalies $(86 \%)(p<0.0001)$ which varied from $77 \%$ for diaphragmatic hernia to $97 \%$ for anencephaly. Subsequent rates of termination following antenatal diagnosis were much higher for DS $(86 \%)$ than the overall rate for FASP9 anomalies $(70 \%)(\mathrm{p}<0.0001)$ with anencephaly being the only individual anomaly to exceed the DS rate $(88 \%)$. The difference in antenatal detection rates between DS and FASP9 cases resulted in a much higher proportion of DS registrations ending in live birth (45\%) compared with FASP9 anomalies $(30 \%)(\mathrm{p}<0.0001)$, despite the higher termination rate. However, neonatal mortality for DS was extremely low $(1 \%)$ compared with $10 \%$ for the FASP9 anomalies $(\mathrm{p}<0.0001)$. 
Table 1 Number of registered cases, antenatal detection rate and outcome of pregnancy by anomaly, 1998-2007

\begin{tabular}{|c|c|c|c|c|c|c|c|c|}
\hline \multirow[b]{2}{*}{ Selected anomalies } & \multirow[b]{2}{*}{ ICD code } & \multirow[b]{2}{*}{$\begin{array}{l}\text { Total } \\
\text { cases }\end{array}$} & \multirow[b]{2}{*}{$\begin{array}{l}\text { Antenatal } \\
\text { detection } \\
\%(\mathrm{n})\end{array}$} & \multirow{2}{*}{$\begin{array}{l}\text { Termination of } \\
\text { pregnancy of those } \\
\text { antenatally } \\
\text { detected \% (n) }\end{array}$} & \multicolumn{4}{|c|}{ Outcome of pregnancy for all cases \% (n) } \\
\hline & & & & & $\begin{array}{l}\text { Termination of } \\
\text { pregnancy }\end{array}$ & $\begin{array}{l}\text { Fetal losses } \\
\text { and stillbirth }\end{array}$ & $\begin{array}{l}\text { Live births } \\
\text { (surviving } \\
>28 \text { days) }\end{array}$ & $\begin{array}{l}\text { Neonatal } \\
\text { death }\end{array}$ \\
\hline Down syndrome (DS) & Q900-9 & 1151 & $57 \%(657)$ & $86 \%(566)$ & $49 \%(566)$ & $6 \%(65)$ & $44 \%(505)$ & $1 \%(15)$ \\
\hline Anencephaly & Q000 & 257 & $97 \%(249)$ & $88 \%(218)$ & $85 \%(218)$ & $8 \%(20)$ & $0 \%(1)$ & $7 \%(18)$ \\
\hline Spina bifida & Q050-Q059 & 339 & $90 \%(303)$ & $78 \%(235)$ & $70 \%(235)$ & $6 \%(20)$ & $22 \%(75)$ & $2 \%(8)$ \\
\hline Hypoplastic left heart & Q234 & 171 & $85 \%(146)$ & $56 \%(82)$ & $48 \%(82)$ & $8 \%(14)$ & $25 \%(42)$ & $19 \%(33)$ \\
\hline Bilateral renal agenesis & Q601/606 & 59 & $81 \%(48)$ & $85 \%(41)$ & $69 \%(41)$ & $15 \%(9)$ & $0 \%(0)$ & $15 \%(9)$ \\
\hline Lethal skeletal dysplasia & Q771-3 & 45 & $82 \%(37)$ & $57 \%(21)$ & $47 \%(21)$ & $7 \%(3)$ & $33 \%(15)$ & $13 \%(6)$ \\
\hline Diaphragmatic hernia & Q790 & 183 & $77 \%(140)$ & $29 \%(40)$ & $22 \%(40)$ & $8 \%(14)$ & $47 \%(86)$ & $23 \%(43)$ \\
\hline Exomphalos & Q792 & 230 & $85 \%(195)$ & $56 \%(109)$ & $47 \%(109)$ & $15 \%(34)$ & $34 \%(77)$ & $4 \%(10)$ \\
\hline Trisomy 18 & Q910-13 & 285 & $85 \%(242)$ & $81 \%(196)$ & $69 \%(196)$ & $13 \%(38)$ & $6 \%(18)$ & $12 \%(33)$ \\
\hline Trisomy 13 & Q914-7 & 127 & $84 \%(107)$ & $83 \%(89)$ & $70 \%(89)$ & $16 \%(20)$ & $5 \%(6)$ & $9 \%(12)$ \\
\hline $\begin{array}{l}\text { All FASP9 selected anomalies } \\
\text { (excl. DS cases) }\end{array}$ & & 1572 & $86 \%(1351)$ & $70 \%(939)$ & $60 \%(939)$ & $10 \%(157)$ & $20 \%(316)$ & $10 \%(160)$ \\
\hline All other registered anomalies & & 10857 & - & - & $9 \%(953)$ & $4 \%(486)$ & $84 \%(9075)$ & $3 \%(343)$ \\
\hline Total registered anomalies & & 13580 & - & - & $18 \%(2458)$ & $5 \%(708)$ & $73 \%$ (9896) & $4 \%(518)$ \\
\hline
\end{tabular}

Poisson regression models (table 2) showed DS registrations were significantly lower in more deprived areas (rate ratio comparing the most deprived with the least deprived tenth: 0.55 $(0.46$ to 0.66$))$. After adjusting for maternal age, no evidence of a trend with deprivation remained (rate ratio (RR) 1.04 (0.86 to $1.25)$ ), with rates similar across the deprivation tenths. This pattern echoed the two FASP9 chromosomal anomalies. For nonchromosomal anomalies, however, the registration rate increased with increasing deprivation (RR 1.41 (1.17 to 1.70)). Overall, there was no evidence of a variation in registration rates with deprivation for the FASP9 anomalies (chromosomal and nonchromosomal combined) (RR 1.05 (0.90 to 1.23)). The fitted models showed no evidence of any two-way interactions.

Similarly, no evidence of variation in antenatal detection rates of the FASP9 anomalies with deprivation emerged (adjusted RR $0.99,95 \%$ CI $(0.83$ to 1.18$))$. This was true for both chromosomal and non-chromosomal FASP9 anomalies (table 2). In contrast, rates of antenatal detection for DS did vary with deprivation (adjusted RR 0.77 (0.58 to 1.01)) being lower in the most deprived areas (45\%) compared with the least deprived $(60 \%)$ although this was not statistically significant. A slightly lower proportion of cases from the least deprived areas was detected before 22 weeks' gestation compared with those from the most deprived areas, though this was not significant (DS: 0.90 (0.69 to 1.19$)$; FASP9: $0.90(0.74$ to 1.08$)$ ).

Following antenatal detection, termination of pregnancy rates varied with socioeconomic deprivation for both DS and the FASP9 anomalies. The difference in termination rates between the most and least deprived areas was similar for both anomaly groups (adjusted RR: DS: 0.77 (0.58 to 1.01$)$; FASP9 anomalies: $0.79(0.64$ to 0.98$))$ and showed little change after adjusting for maternal age. However, in absolute terms, there was a wider gap in termination rates for DS (least deprived 91\%; most deprived 67\%) than for the FASP9 anomalies (least deprived $72 \%$; most deprived 57\%). Rates of stillbirth and fetal loss were high (see table 1) but did not differ significantly for either DS or FASP9 with socioeconomic deprivation (adjusted RR DS: 0.60 (0.27 to 1.35$)$; FASP9: 1.58 (0.94 to 2.66$)$ ).

Consequently, live birth and neonatal mortality rates showed marked socioeconomic variation. For DS, the age-adjusted rate ratio between the most and least deprived cases increased from 1.04 ( 0.86 to 1.25 ) for total registrations to 1.88 (1.42 to 2.49 ) for live births and 6.00 (1.09 to 33.2) for neonatal deaths, although absolute numbers of deaths were small. The FASP9 group exhibited a similar but less marked pattern, with the age-adjusted rate ratio increasing from 1.22 (1.04 to 1.44) for total registrations to 1.85 (1.37 to 2.50$)$ for live births and 2.23 (1.31 to 3.78 ) for neonatal deaths.

\section{DISCUSSION}

Socioeconomic deprivation influences the antenatal detection rate of DS but not the nine severe anomalies screened for by FASP. Some evidence suggests that this inequality arises not only from uptake, but also the offer of screening ${ }^{9}$ with deprivation associated with a lower chance of screening being offered. Once detected, rates of termination of pregnancy were much higher for DS than the FASP9 anomalies, although both groups showed wide socioeconomic differences. These disparities impact on socioeconomic inequalities observed in live births with a congenital anomaly and, for the severe anomalies, the subsequent neonatal mortality. Although there is a significant body of quantitative data investigating the variables associated with the decision to continue or terminate an affected fetus, ${ }^{10-17}$ the complete pathway from antenatal diagnosis through to pregnancy outcome has been sparsely researched, a major difficulty being the lack of detailed, routinely recorded, relevant data.

Antenatal suspicion of a FASP9 anomaly originates either during the first trimester dating scan following pregnancy booking or the fetal anomaly scan, usually conducted between 18 and 21 weeks' gestation. While such scans may represent 'informed' acceptance of screening services, they are widely regarded as an opportunity to date a pregnancy, estimate the date of delivery, visualise and obtain a photograph of the developing fetus ${ }^{18} 19$ and they are frequently considered routine. ${ }^{20-23}$ In contrast, antenatal diagnosis for DS is offered via a defined screening programme involving separate, and conscious, uptake of blood tests and/or invasive procedures.

In the UK, most National Health Service trusts now routinely offer combined first trimester screening for DS and other chromosomal anomalies, including ultrasound measurement of 


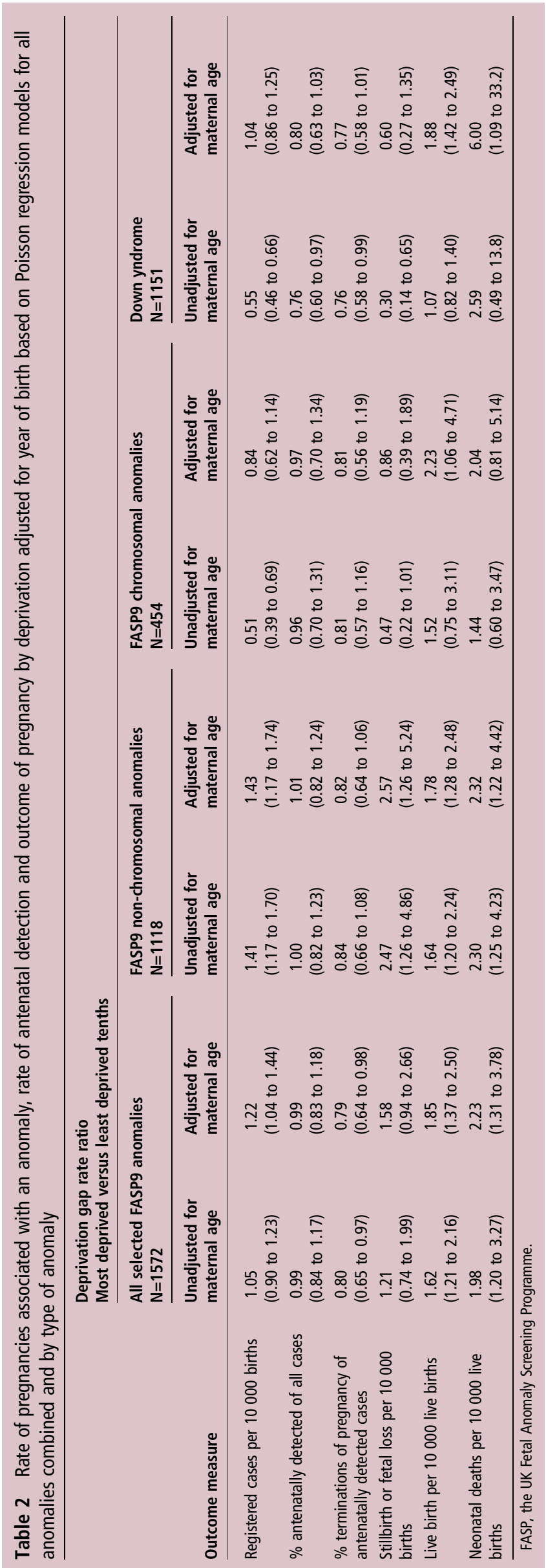

the nuchal thickness of the developing fetus between 11 and 13 weeks. There is some suggestion that access to this relatively new technology, and the earlier diagnosis it facilitates, varies geographically $^{24}$ and is often accessed privately. During the study period, there was inevitable variation between trusts across the region in the precise screening method offered and the implementation of changes, but there is no suggestion that access to prenatal care differed between deprived or more affluent areas. Wide variation in the antenatal detection of DS exists where national screening is offered in Europe, with England and Wales having one of the lowest rates of detection at $66 \%$. In France, this figure has reached 90\%, with Khoshnood et $a l^{25}$ indicating that this has led to a considerable reduction in inequalities.

Rates of pregnancy termination following antenatal detection of DS were much higher than other anomalies, despite DS having near-normal life expectancy, particularly in the absence of any cardiac defect, and the lowest neonatal mortality rate of the selected anomalies. Since it is more prevalent than other anomalies, pregnant women may not only have greater awareness of the condition but their screening uptake may reflect a premeditated decision to respond to a positive anomaly diagnosis by terminating their pregnancy ${ }^{26} 27$ with those women for whom termination is not an option being less likely to enter the screening process. ${ }^{26}$ Attitudes towards learning disability will be relevant, since the clinical likelihood of a live born child surviving with DS is much higher than the FASP9 anomalies.

Rates of termination of pregnancy for all anomalies are lower in more deprived areas. Since later diagnosis is associated with lower rates of termination ${ }^{16} 28$ and evidence suggests a correlation between later presentation and increased deprivation, ${ }^{29} 30$ the options available to women living in more deprived areas may be more restricted should a serious anomaly, particularly DS, be detected. Difficulties in expressing and understanding risk are well documented ${ }^{20}$ with socioeconomic variation in both the communication by health professionals and the interpretation by parents. Evidence suggests that anecdotal reports of women's experiences surrounding the decision to continue a pregnancy after an antenatal diagnosis of a fetal anomaly are supported by real differences in counselling attitudes and procedures between the various clinical professionals subsequently involved. ${ }^{31}$ Such differences are likely to display geographic and socioeconomic variation. Further research is needed to understand whether the socioeconomic variation in rates of termination of pregnancy demonstrated here arises partly from differences in the communication process or the interpretation of risk and subsequent decision making by parents.

\section{Limitations}

Data from antenatal diagnosis through to postnatal outcome are unavailable nationally in the UK, and limited elsewhere to registers belonging to the European Congenital Anomaly Surveillance System (http://www.eurocat-network.eu). Although our data are regional, analysis over a decade, with over 600000 births (around $10 \%$ of the births in England) provides confidence that results are generally applicable elsewhere in the UK and in countries with similar policies on antenatal care and pregnancy termination. While we lack detailed data on individual deprivation measures, possibly attracting problems of confounding, our methods using area-level deprivation data are straightforward and demonstrate how congenital anomaly register data may be used to monitor trends in antenatal detection, termination, live births and mortality.

Only FASP9 conditions where the ICD-10 code uniquely identified an anomaly for which there was a high level of 
certainty about an adverse prognosis were analysed. However, due to a lack of additional information, there still remained variation in prognosis for conditions such as spina bifida and exomphalos. Similarly, due to small numbers, we could not look at specific outcomes for cases exhibiting multiple anomalies. If severity or presence of multiple anomalies was not linked to socioeconomic deprivation, then this would not impact on the findings seen here. Any trend of increasing severity with increasing deprivation would lead to an underestimate of the socioeconomic effect we have identified.

\section{Implications}

Uniform provision of DS screening in the UK is urgently required to reduce socioeconomic inequalities in antenatal detection, which has been achieved elsewhere. ${ }^{27}$ Furthermore, understanding the complex reasons underlying socioeconomic variations in termination rates for congenital anomaly is essential to assess the extent to which they could result from systematic differences in access to services, and communication and interpretation of risk. While the decision to continue an anomaly-affected pregnancy should never be regarded as a flawed choice, an inevitable consequence is an increased concentration of congenital anomalies, particularly DS, together with associated learning difficulties, cardiac problems and other complex health needs, among the most deprived socioeconomic groups. This carries obvious implications for integration of health and social care, and maintaining awareness of these trends is essential to ensure adequate service provision. We therefore urge that existing UK regional anomaly registers be safeguarded, and the current network expanded into areas not presently covered, to permit monitoring of future trends.

Acknowledgements We thank all NHS staff who continue to notify cases to EMSYCAR. Thanks also to Martin Perkins for IT support.

Contributors $J B, E D$ and $L S$ conceived and designed the study. JB and LB were responsible for acquisition of data. RL undertook the initial literature search and LS conducted the statistical analysis. All authors contributed to interpretation of data. $J B$ wrote the first draft of the paper; all authors were involved in subsequent revision and approved the final manuscript. JB is the guarantor.

Funding Until April 2013, EMSYCAR was funded by the Primary Care Trust in the areas covered by the register. LKS was funded by UK Department of Health's NIHR under its Programme Grants for Applied Research (PGfAR) Programme (Grant Reference Number RP-PG-0407-10029). The University of Leicester acted as sponsor of the research. The views expressed are those of the author(s) and not necessarily those of the NHS, the NIHR or the Department of Health.

Competing interests All authors have completed the Unified Competing Interest form at http://www.icmje.org/coi_disclosure.pdf (available on request from the corresponding author) and declare: LKS had financial support from UK Department of Health's National Institute of Health Research for the submitted work; no financial relationships with any organisations that might have an interest in the submitted work in the previous 3 years and no other relationships or activities that could appear to have influenced the submitted work.

Ethics approval The EMSYCAR register is part of the British Isles Network of Congenital Anomaly Registers (BINOCAR) which have ethical approval from MREC (Trent MREC, date 11/09/2009 09/H0405/48) and NIGB (PIAG 2-08(e)/2002 20/06/ 2002) for studies involving the use of their data.

Provenance and peer review Not commissioned; externally peer reviewed.

Data sharing statement No additional data are available from the authors. Aggregated EMSYCAR data may be requested from BINOCAR http://www.binocar. org.uk.

Open Access This is an Open Access article distributed in accordance with the Creative Commons Attribution Non Commercial (CC BY-NC 4.0) license, which permits others to distribute, remix, adapt, build upon this work non-commercially, and license their derivative works on different terms, provided the original work is properly cited and the use is non-commercial. See: http://creativecommons.org/ licenses/by-nc/4.0/

\section{REFERENCES}

1 Oakley L, Maconochie N, Doyle P, et al. Multivariate analysis of infant death in England and Wales in 2005-06, with focus on socio-economic status and deprivation. Health Stat Q 2009(42):22-39.

2 Kurinczuk J, Hollowell J, Boyd $\mathrm{P}$, et al. The contribution of congenital anomalies to infant mortality: inequalities in Infant Mortality Project Briefing Paper 4. Oxford: National Perinatal Epidemiology Unit, University of Oxford, 2010.

3 Kirwan D. NHS Fetal Anomaly Screening Programme: 18+0 to 20+6 Weeks Fetal Anomaly Scan National Standards and Guidance for England. Exeter: NHS Fetal Anomaly Screening Programme, 2010.

4 Smith LK, Budd JL, Field DJ, et al. Socioeconomic inequalities in outcome of pregnancy and neonatal mortality associated with congenital anomalies: population based study. BMJ 2011;343:d4306.

5 Morris J. Congenital Anomaly Statistics 2008: England and Wales. London: British Isles Network of Congenital Anomaly Registers., 2011.

6 Noble M, Wright G, Dibben C, et al. Indices of Deprivation 2004: Report to the Office of the Deputy Prime Minister. London: Neighbourhood Renewal Unit, 2004

7 Lumley T, Kronmal R, Ma S. Relative risk regression in medical research: models, contrasts, estimators, and algorithms. UW Biostatistics Working Paper Series, 2006.

8 Oehlert GW. A note on the delta method. Am Stat 1992;46:27-9.

9 Alderdice F, McNeill J, Rowe R, et al. Inequalities in the reported offer and uptake of antenatal screening. Public Health 2008;122:42-52.

10 Kupek E, Petrou S, Vause S, et al. Clinical, provider and sociodemographic predictors of late initiation of antenatal care in England and Wales. BJOG 2002;109:265-73.

11 Evans MI, Sobiecki MA, Krivchenia EL, et al. Parental decisions to terminate/ continue following abnormal cytogenetic prenatal diagnosis: "what" is still more important than "when". Am J Med Genet 1996;61(4):353-5.

12 Aslan $\mathrm{H}$, Yildirim G, Ongut C, et al. Termination of pregnancy for fetal anomaly. Int J Gynaecol Obstet 2007;99:221-4.

13 Ahmed S, Atkin K, Hewison J, et al. The influence of faith and religion and the role of religious and community leaders in prenatal decisions for sickle cell disorders and thalassaemia major. Prenat Diagn 2006;26:801-9.

14 Tararbit K, Bui TT, Lelong N, et al. Clinical and socioeconomic predictors of pregnancy termination for fetuses with congenital heart defects: a population-based evaluation. Prenat Diagn 2013;33:179-86.

15 Brun JL, Gangbo F, Wen ZQ, et al. Prenatal diagnosis and management of sex chromosome aneuploidy: a report on 98 cases. Prenat Diagn 2004;24:213-8.

16 Chenni N, Lacroze V, Pouet C, et al. Fetal heart disease and interruption of pregnancy: factors influencing the parental decision-making process. Prenat Diagn 2012;32:168-72.

17 Feijen-de Jong El, Jansen DE, Baarveld F, et al. Determinants of late and/or inadequate use of prenatal healthcare in high-income countries: a systematic review. Eur J Public Health 2012;22:904-13.

18 Garcia J, Bricker L, Henderson J, et al. Women's views of pregnancy ultrasound: a systematic review. Birth 2002;29:225-50

19 Lupton D. The Social Worlds of the Unborn. Basingstoke: Palgrave Macmillan, 2013.

20 Pilnick AM, Fraser DM, James DK. Presenting and discussing nuchal translucency screening for fetal abnormality in the UK. Midwifery 2004;20:82-93.

21 Williams C, Sandall J, Lewando-Hundt G, et al. Women as moral pioneers? Experiences of first trimester antenatal screening. Soc Sci Med 2005;61:1983-92.

22 Markens S, Browner $\mathrm{CH}$, Press N. 'Because of the risks': how US pregnant women account for refusing prenatal screening. Soc Sci Med 1999;49:359-69.

23 Chiang HH, Chao YM, Yuh YS. Informed choice of pregnant women in prenatal screening tests for Down's syndrome. J Med Ethics 2006;32:273-7.

24 De Souza E, Alberman E, Morris JK. Down's syndrome: screening and antenatal diagnosis regionally in England and Wales 1989-2008. J Med Screen 2010;17:170-5.

25 Khoshnood B, De Vigan C, Blondel B, et al. Long-term trends for socio-economic differences in prenatal diagnosis of Down syndrome: diffusion of services or persistence of disparities? BJOG 2008;115:1087-95.

26 Liamputtong P, Halliday JL, Warren R, et al. Why do women decline prenatal screening and diagnosis? Australian women's perspective. Women Health 2003;37:89-108

27 Heyman B, Hundt G, Sandall J, et al. On being at higher risk: a qualitative study of prenatal screening for chromosomal anomalies. Soc Sci Med 2006;62:2360-72.

28 Kramer RL, Jarve RK, Yaron Y, et al. Determinants of parental decisions after the prenatal diagnosis of Down syndrome. Am J Med Genet 1998;79:172-4

29 Rowe RE, Garcia J, Davidson LL. Social and ethnic inequalities in the offer and uptake of prenatal screening and diagnosis in the UK: a systematic review. Public Health 2004;118:177-89.

30 Rowe REGJ. Social class, ethnicity and attendance for antenatal care in the United Kingdom: a systematic review. J Public Health Med 2003;25:113-19.

31 Brown SD, Ecker JL, Ward JR, et al. Prenatally diagnosed fetal conditions in the age of fetal care: does who counsels matter? Am J Obstet Gynecol 2012;206:409. e1-11. 